\title{
Outline of Mesoscopic Fluid Mechanics
}

\author{
G. W. $\mathrm{Hu}^{1, *}, \mathrm{X} \cdot \mathrm{S} \cdot \mathrm{Hu}^{2}$ \\ ${ }^{1}$ Aerodynamic Research Institute of Aeronautical Industry of China, P. O. Box 88, Harbin 150001, \\ China \\ ${ }^{2}$ Postgraduate Institute of Harbin Engineering University, Harbin 150001, China \\ Email: cmcw@263.net
}

\begin{abstract}
An Order Conservation System has been put forward in this paper, it consists of 5 Principles on streamline (dynamics). Each streamline has 2 pairs of $\tau$ surface and 4 edges(square section).

Principle 1: In the flowfield every streamline preserves its own fluid lumps, and their mutual order remains unchanged; Principle 2: In shear flow the energy level order (ELO) of every streamline remains unchanged; Principle 3: In shear flow, if a fluid line preserves its lumps all the way, then it must be it streamline; Principle 4: The $\tau$ surface remains the $\tau$ surface, the edge of streamline remains the edge of streamline, the continuity of $\tau$ along the edge and the discontinuity of $\tau$ across the edge remain unchanged; Principle 5: The evolution trend of friction style between two streamlines from sliding to wriggling to rolling can not be reversed.

Based on this system, some results have been obtained: (a) vortex structure and features. loop structure shaped as " $\alpha$ ". over-flow between streamlines, but only occupying part of the loop. streamline? torsion for the streamlines circling in the vortex, the torsion around the streamline (kinematics) is with $180^{\circ}$ positive and $180^{\circ}$ negative. neighborhood of a streamline changes greatly, the number of which is from 4 for the laminar to all streamlines in the vortex. the distinction between the superficial layer and inner layer becomes more obvious than the case in laminar flow pattern, there is no incompressible flow in mesoscopic scale. (b) quantum (Q) state. The parabola profile of velocity of laminar flow on flat plate corresponds to equal level difference of mechanical energy, this case is defined as Quantum state, is of standard spectrum of principle 2,4. (c) expression of shear stress in mesoscopic scale. The shear stress between 2 adjacent streamlines equals the difference of dynamic pressure (vector) of the two $(\tau=\Delta P)$, where $\Delta P$, level difference of dynamic pressure between 2 adjacent streamlines, vector. (d) expression of critical state. The critical state means that the crisis of adhering between 2 adjacent streamlines takes place, its expression is that the maximum value of instantaneous level difference reaches the double of time average level difference $\left((\Delta P)_{\max }=2 \bar{\Delta} P\right)$. the formation of vortex symbolizes the end of critical state. The system of order and the system of quantity just like the two sides of a coin, the reality of one of the two is not less than that of the other. The variety of flow pattern in mesoscopic scale enables the balance of flow pattern in macroscopic scale to be realized. The loop structure of vortex denies the suitability of N-S equation to turbulence.
\end{abstract}

\section{REFERENCES}

1. Hu GW. Deepgoing flow visualization. In: Wei QD, ed. Proceedings of the 4th Asian Symposium on Visualization, 1996; P659-664

2. Hu GW. From N-S equations to flow models. In: Shen X, et al, ed. Modern Techniques and Measurements in Fluid Flows, 1997; ICFDMA, P370-374 